\title{
Investigation of methods for processing materials by magnetoelectric fields
}

\author{
Filippenko Nikolay \\ Transport University \\ Irkutsk, 664075, Russia \\ e-mail: ifpi@mail.ru
}

\author{
Lipchitz Alexandr \\ Transport University \\ Irkutsk, 664075, Russia \\ e-mail: livnet@list.ru
}

\author{
Kargapoltsev Sergey \\ Transport University \\ Irkutsk, 664075, Russia \\ e-mail: popovs@irgups.ru
}

\begin{abstract}
The competitiveness and longevity of machines and mechanisms are a vital task of modern industry. Its solution is based on research of methods that uniquely create a structure with specified physical and mechanical properties. Modern studies of promising methods involve the use of a large number of fields of different nature. Due to this, the goal of this article is the analysis of scientific and practical information in the field of the modification of various materials with the help of electric and magnetic fields. In the paper, the authors argue that the use of fields can significantly increase the energy of ionized particles. As a result, it was found that the effect of electromagnetic waves leads to a reduction and increase in the microhardness of the surface by approximately a factor of 2 , and the rate of growth of the hardened surface layer increases.
\end{abstract}

\section{Keywords-Analysis; Methods; Magnetoelectric}

\section{INTRODUCTION}

Modern studies in the domain of electromagnetic fields and their effects [1-8] confirm that with the help of low-power fields, a direct contactless effect on the structure of the material and the dynamics of its defects at various levels is possible. Such active influence of fields results in a change in the macroproperties of the material and the operational properties of theproduct [22]. In a number of works [9-11], it is noted that processing in electromagnetic fields (EMF) is characterized by low energy costs. Thus, for example, typical energy values reported to one atom in a crystal lattice with some parameter a in commonly used fields are the following magnitudes in sequence:

in a magnetic field $(\mathrm{MF})$

$$
\begin{aligned}
& \Delta \mathrm{W} \approx \mu_{\mathrm{B}} B \approx 10^{-4} \mathrm{eV} \\
& \Delta \mathrm{W} \approx e E a \approx 10^{-4} \mathrm{eV} \\
& \Delta \mathrm{W} \approx j^{2} \rho a^{3} \approx 10^{-4} \mathrm{eV}
\end{aligned}
$$

in an electric field (EF)

in the field of pulse current

in a force field under deformation $\Delta \mathrm{W} \approx E^{\prime} \varepsilon^{2} a^{3} \approx 10^{-3} \mathrm{eV}$ and thermal,

$$
\Delta T=100 K, \Delta W \approx k T \approx 10^{-2} \mathrm{eV}
$$

Here: $\mu$ is the Bohr magneton; $\mathrm{e}$ is the elementary charge; $\rho$ is the specific resistivity; $\mathrm{k}$ is the Boltzmann constant; $\mathrm{E}$ is the energy; E is Young's modulus.

In any real material, defects reduce the degree of its symmetry, locally redistribute the electron density, which causes the emergence of long-range macroscopic electric and magnetic fields [9].

For example, in an ideal material, EF and MF can cause ordering in dipole and magnetic moments, a change in the electronic and phonon spectrum, and phase transitions. As for point defects, although they themselves are rarely the cause of material destruction, an important factor is their interaction with dislocations. For example, if a defect has a dipole or magnetic moment and an elastic anisotropy, its orientation in the EF and MF will cause a decrease in the flow stress in some planes and an increase in the others. In metals, the change in the state of the electron gas in EF and MF (which can be regarded as a set of point stops for dislocations) can affect the plastic properties due to the effect of electron-dislocation interaction. The nature of the interaction of dislocations with fields depends on the nature of the field: the EP can exert a force action on the charged dislocation cores, and the nonuniform $\mathrm{MF}$ can exert a force action on the particles possessing a magnetic moment [9]. Dislocations in various crystals (ionic, covalent, metallic) cause an increased magnetic susceptibility. It is noted [10] that spin-dependent reactions can occur between defects and individual atoms inside the nuclei of dislocations and point complexes. MF changes the multiplicity of the states of the resulting and dissociating pairs, that is, the statistics of these states in the material.

As for the effect of EMF on macrodefects and the sample as a whole, the difference in the density of EMF energy in adjacent elementary volumes of the sample or on the surface causes the emergence of electrodynamic forces, and the flowing current causes warming up, which leads to the emergence of thermal influences and stresses.

In addition, a number of related phenomena that have been reduced to a significant influence of MF on various physical 
and mechanical and other structural-sensitive characteristics of nonmagnetic materials have been found. Constant, variable, pulsed and microwave fields have been used, practically all therepertoire of microstructure and mechanical properties' studies (Fig. 1), various types of loading and temperature ranges.

Great attention was immediately paid to the work of V.I. Alshits with his colleagues [11]. It described a contradictory phenomenon, at first glance: the stimulation of the motion of dislocations in $\mathrm{NaCl}$ single crystals, introduced by a weak impact, a constant MP. Further studies in this area have shown that the magnetoplastic effect leads to a decrease in the yield point, a decrease in the microhardness and internal friction of various single-crystal materials.

According to [11], effects (EMF) can be divided into three groups with respect to time characteristics: those that manifest themselves during their impact, as well as reversible and irreversible effects that have a long aftereffect (Fig. 2). It is assumed that the non-equilibrium in the structure (crystal) ensures high sensitivity of the defect structure to the effect of external and internal EMF.

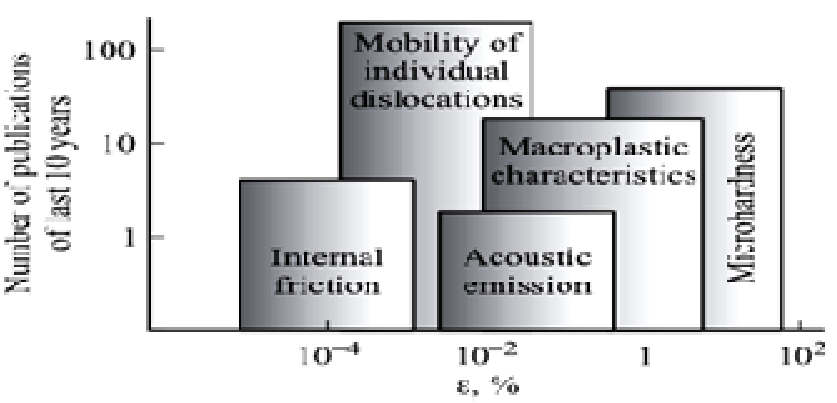

Fig. 1. Number of registered publications on MEP for a decade, affecting different ranges of relative strain

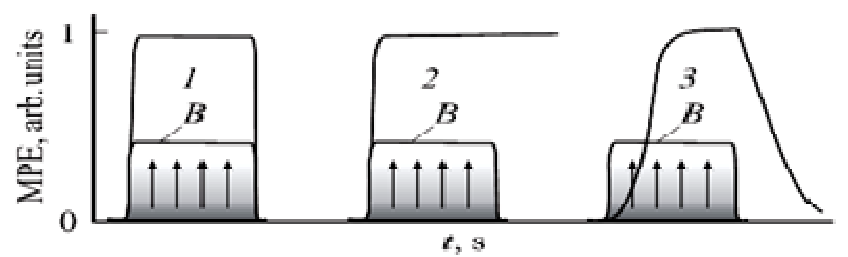

Fig. 2. Three main types of behavior of non-magnetic materials in MEP: 1 - in situ effects arising from the action of EMF, 2 - irreversible post-effects, 3 aftereffect with slow relaxation and latent period after disconnection

In studies [10,13-14] investigations of the effect of a weak constant magnetic field on the collision of an electron with scattering centers on the mechanical properties of various nonmagnetic crystals ( $\mathrm{NaCl}, \mathrm{CsI}$, etc.) and metals $(\mathrm{Zn}, \mathrm{Al})$ were carried out. In experiments with nonmagnetic crystals, the motion of dislocations was induced by a magnetic field with $\mathrm{B}=0.1 \ldots 1.6 \mathrm{~T}$ without additional mechanical loading. In these studies it is noted that with an increase in the exposure time of the samples in the MF, the mean dislocation path length $\mathrm{L}$ grew linearly, and when the temperature was varied, the path length $\mathrm{L}$ practically did not change. When the sign of the field is reversed, the direction of dislocation motion did not change, so the authors excluded the possibility of explaining the effect by the action of ponderomotive forces or a vortex electric field. It is proved [6] that the MF lowers the height of potential barriers for the motion of dislocations, and the driving force for dislocation transport is a random mosaic of internal stress fields. It is assumed that MF affects the interaction of moving dislocations with impurity centers and the internal structure of both. Analysis of the dependence of the average velocity of motion of edge dislocations on the distance covered in $\mathrm{NaCl}$ crystals, untreated and processed in MF, showed that MF induced the aftereffect: it facilitated the movement of dislocations after the field was turned off. Investigation of the influence of $\mathrm{MF}$ with $\mathrm{B}=0.2 \mathrm{~T}$, which was applied when the indenter was introduced into bismuth crystals, showed that microhardness and the size of the twin layers around the imprint were observed to increase [15]. The opposite results were obtained on aluminum [16, 17], it was established that holding in a magnetic field reduces the microhardness of aluminum, and there exists a threshold value for the induction of a constant magnetic field, from which the effect of a linear decrease in the microhardness is observed. It is found that creep under conditions of an external magnetic field results in a decrease in the average size of the pits of a ductile fracture by more than 2 times and contributes to an increase in the scalar density of dislocations that form the substructure of dislocation chaos. This indicates an increase in the degree of attachment of dislocations and an increase in the ductility of the material.

\section{PHYSICS OF ELECTROMAGNETIC FIELDS AND THEIR INFLUENCE ON MATERIAL PROPERTIES}

Experimental studies of the change in the strength characteristics of samples of PA-66 polyamide in highfrequency $\mathrm{EMF}$ in [18] were made in accordance with regulatory requirements. After specially simulated operating conditions, corresponding to the real conditions of Western and Eastern Siberia, the samples were placed in the EMW field for a short time.

The results of full-scale tests are shown in the diagrams (Fig. 3, 4).

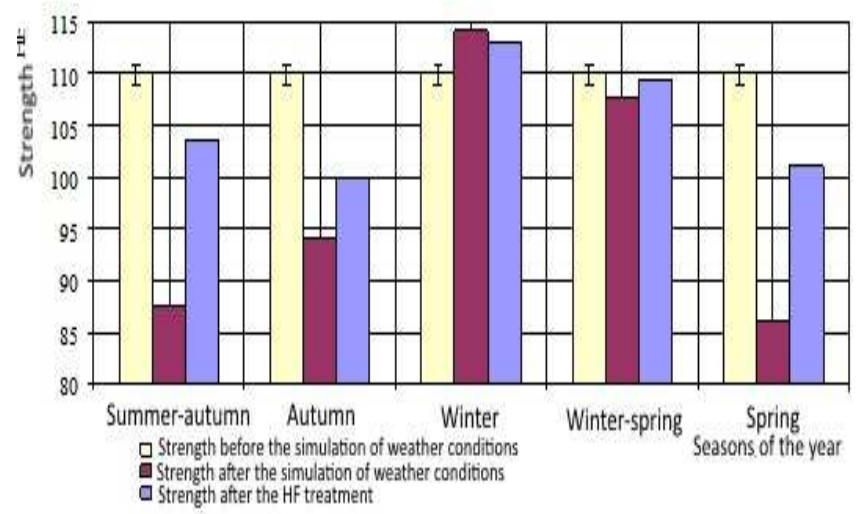

Fig. 3. Results of hardness measurements of samples 


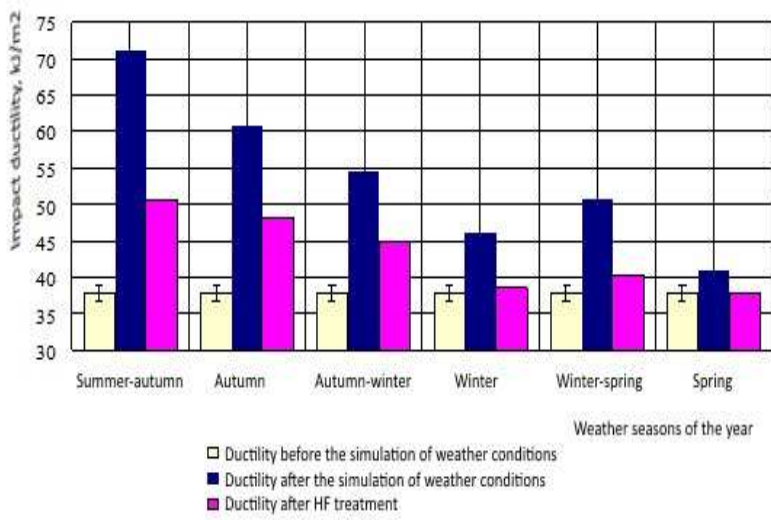

Fig. 4. Results of measurements of impact ductility of samples

The presented review of literature sources indicates the complexity. Analysis of the obtained data proves that the strength characteristics of PA-66 polyamide samples, after HF treatment, in some cases exceeded their original values.

In [19], the authors propose a method for ionizing nitriding instrumental steels in crossed electric and magnetic fields. It is argued that the use of fields can significantly increase the energy of ionized particles. As a result, it was found that the effect of EMF results in an increase in the microhardness of the surface about 6 times, and the rate of growth of the hardened surface layer increases.

In [20], the authors propose a method for softening the materials of the crystal structure, which involves the action of the main and additional EMF, the lines of the additional magnetic field directed at an angle of $30-90{ }^{\circ}$ to the lines of force of the main field, the pulse duration was 10-2 to $10-9 \mathrm{~s}$. The achieved softening result in this case is associated with the following: an external magnetic field affects a material containing magnetic grains (magnetite) or minerals with a piezoelectric effect (quartz), causing the effect of magnetostriction and (or) piezostriction in these grains. Also, because of the heterogeneity of the properties of the materials that make up the sample and the presence of water in the micropores, stresses emerge at the grain boundaries. That is, over the entire volume of the material, stretching and shearing stresses arise, resulting in the destruction of the material.

Thus, relying on the arguments of the presented papers, it can be concluded that the effect of force fields on materials is reduced to the following:

\section{Multifaceted impact of EMF on various materials.}

2. The emerging macroscopic effects of EMF influence are the result of influence on all structural and hierarchical levels of the material.

3. There is a threshold character of post-effects of MF effect and saturation of MPE

4. Over the magnetic field, which indirectly indicates a selective effect on certain defects in the crystal, i.e. MF changes the ratio of the factors influencing physical and mechanical properties in a special way.

5. EMF occurs in non-equilibrium structures.
6. There are several channels of influence of EMF on the structure and properties of the material. It is assumed that EMF induces multistage relaxation processes of the defect structure. A part of these processes (depinning of dislocations, disintegration of impurity complexes and subsequent recombination of disintegration products on other structure defects and among themselves) is unique and cannot be initiated by such traditional influences as thermal treatment, mechanical impact, etc.

7. Strong and weak EMF influences various objects in the material.

8. EMF causes a change in the properties of materials of different magnetic nature, which makes it possible to use EMF treatment to modify both magnetic and nonmagnetic materials.

9. Regulation and selection of processing modes in EMF allows solving specific scientific and production tasks, to obtain a given set of properties.

\section{CONCLUSION}

1. The results of intensive studies of MPEs in recent years have shown the reality of the effects of a significant influence of weak EMF on the mechanical properties of many materials. This opens the potential for investigating not only the conditions, regularities and nature of the influences themselves, but also a wider range of multi-stage processes in the system of structural defects. All this creates a foundation for a radically new and highly sensitive method of electromagnetic spectroscopy of fast-paced phenomena in real time. At the same time, for an adequate description of the physics of the process in a number of materials, it is necessary to consider electronic processes at a new electron-spin and even spin-nuclear level.

2. In addition to the principle significance, where for the first time direct methods have shown the important role of structural defects in the formation of the mechanical properties of nonmagnetic materials and their sensitivity to EMF, the described results can be useful in related fields of knowledge [21]. So, for example, HF treatment can in some cases replace a much longer and energy-intensive heat treatment conducted for the accelerated relaxation of the defective structure and its stabilization in various materials. The study of the regularities and physical mechanisms of the influence of weak fields on various properties of solids can be considered as part of the large problem of the effect of small doses (irradiation, chemical action, electromagnetic fields, etc.) on open nonequilibrium systems in living and inanimate nature, and itself such influence - as an important factor of the environment. For example, geodynamics and tectonics should apparently take into account the potential weakening of the crustal materials as a result of the combined effect of the permanent EMF of the Earth and its high-frequency fluctuations. Probably, both magnetobiology and biochemistry can borrow reasoning patterns and physical models that have been worked out and tested on simpler crystalline and amorphous systems. In one way or another, the study of the effect of EMF already gave impetus to the study of other electromagnetically sensitive properties (electrical, optical, luminescent) in diamagnetic 
materials of great practical importance (semiconductors, polymers, fullerites, high-spin organic compounds [21], etc.).

3. The present research does not claim to be complete (if only because of the limited volume), but aims to further study the remarkable and most reliable data obtained in recent years by several independent scientific groups.

\section{References}

[1] Yu.A. Osipyan, (ed.),Electronic properties of dislocations in semiconductors.URSSEditorial board, Moscow, 2000, $320 \mathrm{p}$.

[2] Yu.A. Klimov, V.M. Maslovsky, V.V. Tarasenko, Electrical engineering Vol. 3, 5 (139), pp. 25, 1990.

[3] Yu.A. Klimov, V.M. Maslovsky, K.V. Kholodnov, Electricalengineering, Vol. 3, 5 (144), pp. 22, 1991.

[4] A.G. Kadmensky, S.G. Kadmensky, M.N. Levin, V.M. Mazosky, A.V. Chernyshev, Letters to the ZhTF, Vol. 19, 3, pp. 41, 1993.

[5] M.N. Levin, Yu.O. Lichmanov, V.M. Maslovsky, Letters to the ZhTF Vol. 20, 4, pp. 27, 1994.

[6] K.M. Salikhov, Y.N. Molin, R.A. Sagdeev, A.L. Buchachenko, Spin Polarization and Magnetic Effects in Radical Reactions. Elsevier, Amsterdam, 1984, 415 p.

[7] A.L. Buchachenko, R.Z. Sagdeev, K.M. Salikhov, Magnetic and spin effects in chemical reactions. Nauka Publ., Novosibirsk, 1978, 296 p.

[8] A.L. Buchachenko, Chem. Rev, Vol. 95, 7, pp. 2507, 1995.

[9] Yu.I. Golovin "Electromagnetic Aspects of Strength and Plasticity Physics of solid bodies," Bulletin of Tambov University. Ser. Natural and technical sciences, Vol. 1, No. 1. pp. 3-20, 1996.

[10] V.I. Alshits, E.V. Darinskaya, T.M. Perkalina, A.A. Urusovskaya, "The motion of dislocations in $\mathrm{NaCl}$ crystals under the action of a constant magnetic field," Solid State Physics, Vol. 29, No. 2, pp. 467-470, 1987.
[11] Yu.I. Golovin, "Magnitoplasticity of Solids (Review)," Physics of the Solid State, Vol.46, No. 5, pp. 769-803, 2004.

[12] V.I. Alshits, E.V. Darinskaya, E.A. Petrzhik, "In situ study of the magnetoplastic effect in $\mathrm{NaCl}$ crystals by the method of continuous etching," Physics of the solid, Vol. 33, No. 10, pp. 3001-3010, 1991.

[13] V.I. Alshits, E.V. Darinskaya, E.A. Petrzhik "Magnetoplastic effect in CsL and LiF crystals," Solid state physics, Vol. 35, No. 2, pp. 320-322, 1993.

[14] Yu.I. Golovin, R.B. Morgunov, S.E. Zhulikov, "The role of internal mechanical stresses in magnetically-stimulated motion of dislocations," Crystallography, Vol. 43, No. 4, pp. 689-693, 1998.

[15] N.G. Filippenko, S.I. Popov, A.V. Livshits, "Experimental research of the possibility of restoration of products from polymeric materials," Transport infrastructure of the Siberian region: Materials of the third All-Russian scientific and practical conference with international participation, May 13-17, 2013. Irkutsk: ISTU, pp. 430-437, 2013.

[16] K.N. Ramazanov, V.V. Budilov, R.K. Vafin, "Nitriding of high-speed steel P6M5 in a glow discharge with superposition of a magnetic field," Hardening technologies and coatings, No. 5, pp. 39-42, 2010.

[17] P.P. Ananiev, S.A. Goncharov, E.L. Belchenko, V.P. Stupnikov, A.A. Ostashevsky, I.A. Ter-Gukasov, V.A. Morozov, S.I. Botva, Method of softening of materials of the crystal structure and the device for its implementation, Patent EA 003853B1: IPC B 02 C 19/18 E 21 C 37/18 C 22 B 3/22.

[18] Yu.I. Golovin, Review of papers on the investigation of the influence of weak magnetic fields Derzhavin Tambov State University. 392622 Tambov, Russia.

[19] T.G. Prokhorova, S.S. Khasanov, L.V. Zorina, L.I. Buranov, V.A. Tkacheva, A.A. Baskakov, R.B. Morgunov, M. Gener, E. Canadell, R.P. Shibaeva, E.V. Yagubskii, Adv. Funct. Mater., Vol. 13, 5, 1, 2003.

[20] A.V. Komshina, A.S. Pomelnikova, "Prospectivity of the method of low-energy processing of materials using a magnetic field," Science and Education El, Vol. FS 77, pp. 48211, September 2012. 